\title{
Ocular imaging at the cutting-edge
}

\author{
Timothy Y. Y. Lai $\mathbb{D}^{1,2}$
}

Received: 23 October 2020 / Revised: 28 October 2020 / Accepted: 28 October 2020 / Published online: 11 November 2020

(c) The Royal College of Ophthalmologists 2020

Barely three decades ago, ophthalmic images were mostly limited to film-based slit-lamp photography, fundus photography, fluorescein angiography (FA) and B-scan ultrasounds. With the rapid development in digital imaging technology and computer processing power, multiple innovative ocular imaging investigations have become available and are now essential parts of our daily clinical practice. In this special issue of Eye, experts from around the world summarised the recent major advancements in ocular imaging and their impacts in various fields of ophthalmology.

One of the most remarkable development in the field of ocular imaging is the optical coherence tomography (OCT). OCT has greatly enhanced our ability to visualise both the anterior and posterior segments of the eye and is now part of the standard-of-care in the management of macular diseases and glaucoma. OCT-based parameters have also become the standard biomarkers in clinical trials for retinal diseases [1]. The concurrent availability of OCT and intravitreal antivascular endothelial growth factor therapy for macular diseases has also enabled OCT-guided personalised treatment which has led to favourable visual outcomes with considerable cost savings [2]. Current generations of spectral-domain OCT (SD-OCT) and swept-source OCT (SS-OCT) machines enable high resolution images to be captured at much faster speeds with wider field of view compared with the previous generations of time-domain OCT. Increase in acquisition speed has also allowed the detection of flow signals for OCT angiography (OCTA). Compared with dye-based angiography, OCTA has the advantage of being non-invasive and allows the

Timothy Y. Y. Lai

tyylai@cuhk.edu.hk

1 Hong Kong Eye Hospital, Department of Ophthalmology \& Visual Sciences, The Chinese University of Hong Kong, Kowloon, Hong Kong

22010 Retina \& Macula Centre, Kowloon, Hong Kong evaluation of individual retinal and choroidal vascular layers both cross-sectionally and en face. There has been a large amount of recent publications in the use of OCTA for evaluating various retinal diseases including diabetic retinopathy and neovascular age-related macular degeneration (AMD) and some of these findings are summarised in the articles by Querques et al. [3], Corvi et al. [4], and Sun et al. [5].

The use of multimodal imaging with OCT, OCTA, FA, indocyanine green angiography (ICGA) and fundus autofluorescence (FAF) has greatly enhanced our ability to evaluate posterior segment diseases. In the article by Liu and Moore [6], the recent findings on congenital focal abnormalities of the retina and retinal pigment epithelium were discussed. SD-OCT and FAF enabled better characterisation of these developmental lesions in vivo. In the articles by Tugal-Tutkun et al. and Herbort et al. [7, 8], the authors summarised the advances and potential new developments in using multimodal imaging in the evaluation of posterior uveitis. Investigations such as enhanceddepth imaging OCT (EDI-OCT), SS-OCT, OCTA and ICGA have been found to be particularly useful for assessing choroidal inflammatory involvement in posterior uveitis. New insights gained from the evaluation of choroidal vasculature by ICGA and EDI-OCT have also led to description of new disease entities like pachychoroid eye diseases [9]. A specific form of drusenoid lesion which is commonly seen in eyes with pachychoroid diseases has been labelled as pachydrusen and the concept and clinical significance of pachydrusen and other forms of drusen are discussed in the article by Zhang and Sivaprasad [10].

The introduction of deep learning (DL) in artificial intelligence (AI) and the concept of "big data" has revolutionised the field of $\mathrm{AI}$ and $\mathrm{AI}$ is now frequently referred to as the "fourth industrial revolution" [11]. Due to the large amount of data generated from various ocular imaging investigations, AI with DL can be applied for various diagnostic evaluations, particularly in diabetic retinopathy, AMD and glaucoma [12-14]. In the article by Ran et al. [15], the authors provided an overview on recent studies 
which utilised DL of OCT findings in the evaluation of glaucoma and how it can potentially be deployed in the clinical setting.

Most of the existing ocular imaging technologies are generally used for assessing anatomical or structural changes. With the modification of existing technology, functional imaging of metabolic activity has now become a possibility. Chen et al. summarised the recent development in a non-invasive imaging technique which measures retinal flavoprotein fluorescence (FPF) [16], which can act as a biomarker for mitochondrial activity. Oxidative stress occurs in diseases such as diabetic retinopathy, AMD and glaucoma and is a major cause of mitochondrial dysfunction. Therefore, measuring FPF might be helpful in evaluating these conditions. Imaging techniques can also be adapted for assessing the structure and/or function of the aqueous outflow pathways. In the article by Lusthaus et al. [17], the authors described various non-invasive imaging techniques including anterior segment OCTA, phase-sensitive OCT and haemoglobin video imaging which allow analysis of aqueous outflow under physiological conditions.

Improvement in imaging technology has also brought down the cost of many existing devices. Rajalakshmi et al. reviewed different types of fundus cameras which are used for diabetic retinopathy screening worldwide [18]. Several smart phone-based fundus camera devices have already been approved by the US Food and Drug Administration for retinal imaging and some of these devices can even incorporate AI-based imaging analysis. With increasing availability of these affordable fundus cameras, it will hopefully provide better access of care and reduce the global burden of eye diseases.

Despite all the recent advancements and innovations in ocular imaging, there are still some outstanding questions which should be discussed and addressed within the ophthalmic community. One fundamental issue concerns the training curriculum of future ophthalmologists. The extent of how these imaging technologies will impact the basic clinical skills of our residents and fellows remains to be seen. Another issue is how to determine the optimal strategy for incorporating these rapidly-evolving ocular imaging technologies into clinical guidelines. Since many of the imaging instruments are rather expensive, the extent and timing of adopting these new technologies may also need to be considered carefully and will depend on resource availability, clinical expertise, workload and economy of scales. Health economic evaluations such as cost-effective and cost-utility analyses will be useful in assisting stakeholders to make informed decisions in planning for imaging services. The implementation of AI-induced "black-box" ocular imaging will also change the future roles of clinicians and the patients' experience, resulting in complex ethical issues in areas of patient privacy, confidentiality, safety, autonomy and beyond [19]. Nonetheless, regardless of the potential changes which might be caused by AI, ophthalmologists should still play a central role as the key human element in the delivery of care.

\section{Compliance with ethical standards}

Conflict of interest TYYL has received honoraria for consultancy, lecture fee and grant support from Allergan, Bayer, Novartis and Roche.

Publisher's note Springer Nature remains neutral with regard to jurisdictional claims in published maps and institutional affiliations.

\section{References}

1. Phadikar P, Saxena S, Ruia S, Lai TY, Meyer CH, Eliott D. The potential of spectral domain optical coherence tomography imaging based retinal biomarkers. Int J Retin Vitreous. 2017;3:1.

2. Windsor MA, Sun SJJ, Frick KD, Swanson EA, Rosenfeld PJ, Huang D. Estimating public and patient savings from basic research - a study of optical coherence tomography in managing antiangiogenic therapy. Am J Ophthalmol. 2018;185:115-22.

3. Querques G, Borrelli E, Battista M, Sacconi R, Bandello F. Optical coherence tomography angiography in diabetes: focus on microaneurysms. Eye. 2020. https://doi.org/10.1038/s41433-02001173-7.

4. Corvi F, Su L, Sadda SR. Evaluation of the inner choroid using OCT angiography. Eye. 2020. https://doi.org/10.1038/s41433020-01217-y.

5. Sun Z, Yang D, Tang Z, Ng DS, Cheung CY. Optical coherence tomography angiography in diabetic Retinopathy: an updated review. Eye. 2020. https://doi.org/10.1038/s41433-020-01233-y.

6. Liu Y, Moore AT. Congenital focal abnormalities of the retina and retinal pigment epithelium. Eye. 2020;34:1973-88.

7. Tugal-Tutkun I, Herbort CP Jr, Mantovani A, Neri P, Khairallah M. Advances and potential new developments in imaging techniques for posterior uveitis. Part 1: noninvasive imaging methods. Eye. 2020. https://doi.org/10.1038/s41433-020-1063-1.

8. Herbort CP Jr, Tugal-Tutkun I, Mantovani A, Neri P, Khairallah M, Papasavvas I. Advances and potential new developments in imaging techniques for posterior uveitis Part 2: invasive imaging methods. Eye. 2020. https://doi.org/10.1038/s41433-020-1072-0.

9. Cheung CMG, Lee WK, Koizumi H, Dansingani K, Lai TYY, Freund KB. Pachychoroid disease. Eye. 2019;33:14-33.

10. Zhang X, Sivaprasad S. Drusen and pachydrusen: the definition, pathogenesis and clinical significance. Eye. 2020. In press.

11. Cheng CY, Soh ZD, Majithia S, Thakur S, Rim TH, Tham YC, et al. Big data in ophthalmology. Asia Pac J Ophthalmol. 2020; 9:291-8.

12. Mayro EL, Wang M, Elze T, Pasquale LR. The impact of artificial intelligence in the diagnosis and management of glaucoma. Eye. 2020;34:1-11.

13. Grzybowski A, Brona P, Lim G, Ruamviboonsuk P, Tan GSW, Abramoff M, et al. Artificial intelligence for diabetic retinopathy screening: a review. Eye. 2020;34:451-60.

14. He M, Li Z, Liu C, Shi D, Tan Z. Deployment of artificial intelligence in real-world practice: opportunity and challenge. Asia Pac J Ophthalmol. 2020;9:299-307.

15. Ran AR, Tham CC, Chan PC, Cheng CY, Tham YC, Rim TH, et al. Deep learning in glaucoma with optical coherence 
tomography: a review. Eye. 2020. https://doi.org/10.1038/s41433020-01191-5.

16. Chen AX, Conti TF, Hom GL, Greenlee TE, Raimondi R, Briskin IN, et al. Functional imaging of mitochondria in retinal diseases using flavoprotein fluorescence. Eye. 2020. https://doi.org/10. 1038/s41433-020-1110-y.

17. Lusthaus JA, Khatib TZ, Meyer PAR, McCluskey P, Martin KR. Aqueous outflow imaging techniques and what they tell us about intraocular pressure regulation. Eye. 2020. https://doi.org/10. 1038/s41433-020-01136-y.

18. Rajalakshmi R, Prathiba V, Arulmalar S, Usha M. Review of retinal cameras for global coverage of diabetic retinopathy screening. Eye. 2020. In press.

19. Rigby MJ. Ethical dimensions of using artificial intelligence in health care. AMA J Ethics. 2019;21:E121-4. 